> Le nombre des médecins n'a jamais été aussi important en France. Pourtant, certains départements manquent de plus en plus de médecins généralistes et les « déserts médicaux » ne semblent pas près de disparaître, bien au contraire. La situation du médecin généraliste en milieu rural, vénéré au XIXe et au début du XXe siècle, et toutes ces grandes figures de praticiens faisant à cheval leurs visites, est devenue totalement désuète. Les malheureux qui continuent d'exercer dans ces régions en déclin aux populations vieillissantes ont des gardes trop nombreuses et des rythmes de travail déments, ils manquent de plateaux techniques qui pourraient leur simplifier la vie; ils ne peuvent trouver de remplaçant, que ce soit pour se recycler ou partir en vacances, et ils ne se font guère d'illusions: aucun jeune médecin n’acceptera de leur succéder. L'épuisement conduit parfois même au suicide. Dans certaines régions, les collectivités locales se résignent à recruter des médecins étrangers: ceux-ci s'adaptent avec plus ou moins de bonheur dans ces pays qui ont conservé de fortes particularités locales. À l'heure où l'écologie devient primordiale, où la pollution et les conditions de vie dans les grandes villes (transports, logements, insécurité...) sont unanimement dénoncées, il est paradoxal de constater que la densité de population dans ces zones urbaines continue à augmenter. Ce phénomène n'est pas propre à la France: on le retrouve de façon plus aiguë encore dans les pays en voie de développement; il existe aussi en Europe (en Allemagne en particulier), de même qu'au Québec, où le Collège des médecins, en janvier 2007, vient de faire le point sur ce problème du manque de médecins de famille. Quelles sont les solutions proposées pour y remédier? C'est ce que nous tenterons de définir. <

\section{Démographie médicale}

Comment lutter contre

les déserts médicaux?

François Flori, Simone Gilgenkrantz
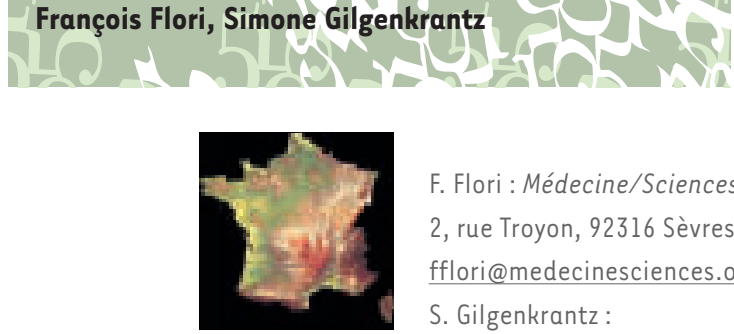

F. Flori : Médecine/Sciences, EDK, 2, rue Troyon, 92316 Sèvres Cedex, France. fflori@medecinesciences.org

S. Gilgenkrantz :

Médecine/Sciences, 9, rue Basse, 54330 Clérey-sur-Brénon, France. sgilgenkrantz@medecinesciences.org

\section{État des lieux}

En France, en 2005, on comptait 104556 omnipraticiens (toutes activités confondues) et 101308 spécialistes. La densité moyenne pour 100000 habitants serait donc de 340 médecins, dont 167 médecins généralistes. Mais le nombre de médecins effectivement actifs est moindre. Une étude parue dans médecine/sciences en 2001 avait déjà analysé les diverses orientations des médecins généralistes [1]. En 2002, le nombre de médecins libéraux généralistes était de 70000 et celui des médecins libéraux spécialistes de 62000 . Selon les projections de la DRESS (Direction de la Recherche, de l'Évaluation, des Études et des Statistiques) [2], ces chiffres devraient baisser avec un recul de $9 \%$ de libéraux généralistes en 2025 et globalement, le nombre de médecins diminuerait d'environ $15 \%$ pour atteindre (toujours pour 100000 habitants) 283 médecins en moyenne en 2025 (Figure 1).

\section{Prévision d'une baisse du nombre des médecins}

Les raisons en sont multiples:

- La moyenne d'âge est de 48 ans chez les médecins généralistes, et de nombreux médecins vont prendre leur retraite.

- Les promotions ont diminué à partir de l'instauration en 1971 d'un numerus clausus à la fin de la $l^{\text {re }}$ année des études de médecine.

Ce numerus clausus a fait diminuer le nombre des étudiants en médecine (3 500 en 1992-1993 pour remonter à 6200 en 2004-2005). II a eu aussi un retentissement sur les inscriptions en médecine, le concours étant considéré comme trop difficile, et d'autres professions (commerce, écoles d'ingénieurs), semblant plus attractives chez les jeunes. La dernière décision ministérielle augmente le numerus clausus à 7000 pour l'année 2006-2007, ce qui aura pour effet d'augmenter le nombre de médecins dans 10 ans (étant donné la durée des études de médecine), et peut-être d'attirer plus d'inscriptions dans les facultés de médecine.

- Une augmentation relative de la population se poursuivra pendant cette période. 
Les médecins choisissent de préférence des spécialités

Une étude faite en 2001 montrait clairement que le nombre d'omnipraticiens est inférieur au nombre de spécialistes. De plus, il existe parmi les omnipraticiens de nombreux modes d'exercice répertoriés ou non (homéopathie, acupuncture, ostéopathie...) très éloignés de la médecine générale en ambulatoire [3]. Les résultats de l'ECN (examen national classant) montre nettement que chaque année un pourcentage important de postes d'internes en DES (diplôme d'études spécialisées) de médecine générale ne sont pas pourvus. Certains s'orientent vers divers secteurs (prévention, administration). Parmi ceux qui exercent véritablement la médecine générale, de plus en plus se dirigent vers des cabinets de groupe et des activités liées à des secteurs hospitaliers.
Et pour finir, les médecins généralistes exerçant en cabinet individuel se trouvent essentiellement en région parisienne $(21 \%)$ et dans les villes de province de plus de 200000 habitants (26\%).

En 2006, le nombre de médecins généralistes libéraux actifs est évalué à 68411 ; on mesure la différence avec le nombre d'omnipraticiens [4].

\section{La féminisation de la profession médicale} fait diminuer le nombre des généralistes

Les femmes représentent $38,8 \%$ des médecins en France métropolitaine. Elles s'orientent plus souvent vers des spécialités. La proportion des femmes est d'autant plus forte que les classes d'âge sont jeunes (Figure 2). Parmi les étudiants inscrits en première année en 2004, 64 \% sont des femmes. Même si elles optent pour la médecine générale, le choix du cabinet individuel est peu fréquent. Installées en médecine libérale, elles ont une activité moyenne inférieure, c'est-à-dire égale à $70 \%$ de celle de leurs collègues masculins. Elles ne bénéficient pas des mêmes avantages que les salariées quand elles sont enceintes et ne sont pas dispensées de garde en fin de grossesse. Le SNJMG (syndicat des jeunes médecins généralistes) a attiré l'attention des pouvoirs publics sur ce sujet.

Figure 1. Effectifs de médecins (moins de 80 ans) de 1995 à 2025 pour la France (sources: Adeli redressé). Projections DREદS ( $\mathrm{nc}=7000)$.

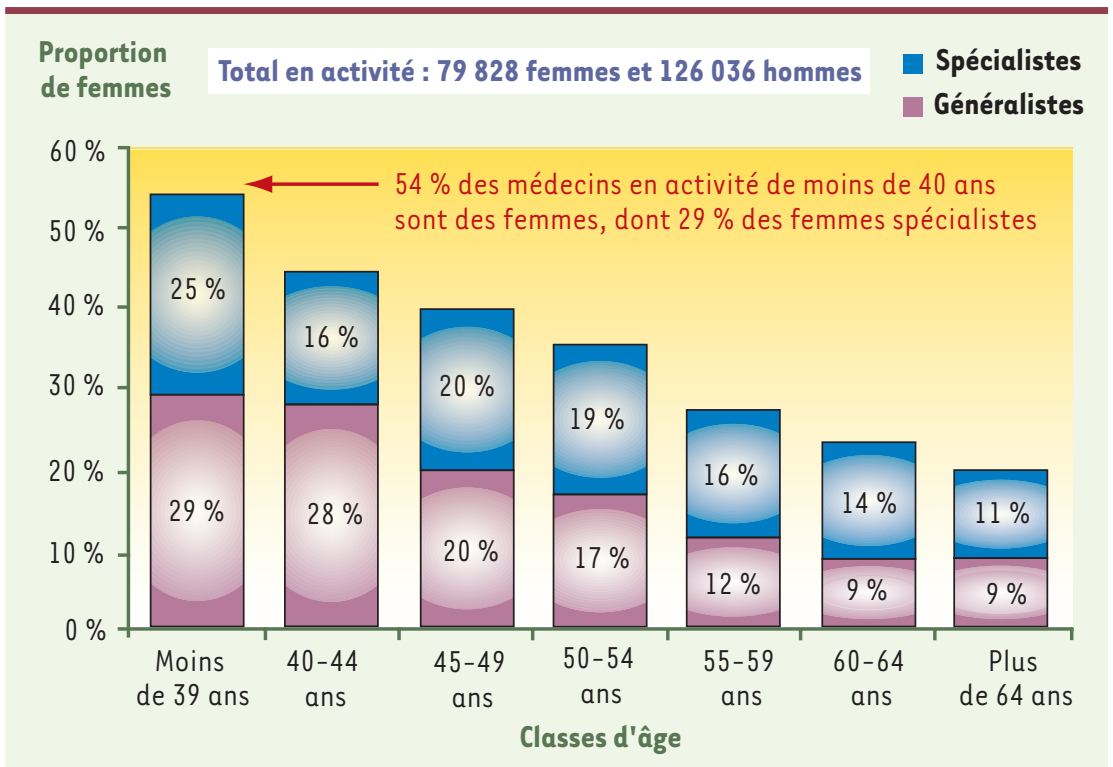

Figure 2. Proportion des femmes selon l'âge et la répartition généralistes/spécialistes de chaque tranche d'âge (pour le total des médecins en métropole) (source CNOM 2006).

\section{Le désir d'un temps}

de travail allégé est fréquent

parmi les femmes et les jeunes

Les nouvelles générations de médecins refusent les horaires trop élevés du généraliste qui interdisent une vie de famille normale avec participation active à l'éducation des enfants. La perspective d'une installation en cabinet de groupe est privilégiée, de même que des activités diverses pour éviter le surcroît de gardes sont les principaux acteurs des soins de proximité, en première ligne pour le suivi des grossesses, des nourrissons, et de la contraception. Ils souhaitent donc aussi être proches d'un hôpital afin de pouvoir disposer rapidement d'un plateau technique. C'est pourquoi, en cas d'installation individuelle, celle-ci se fait le plus souvent dans les grandes villes. et des horaires trop lourds. Ces médecins 
La répartition de l'ensemble des médecins sur le territoire

Passer dix ans d'études pour devenir médecin dans une grande ville, fonder une famille et avoir des enfants n'incitent pas les jeunes médecins à s'installer en milieu rural, surtout si l'épouse a elle-même une profession, et si les collèges et lycées sont trop distants. De plus, chaque fois qu'ils peuvent choisir après l'દCN (examen classant ayant remplacé l'internat), ils optent pour des régions agréables (sur le plan du climat et des activités culturelles : Île-de-France, région PACA).

C'est pourquoi le gradient nord-sud est très net.

Les régions les mieux dotées sont la région PACA et la région bordelaise. La région parisienne paraît moins bien dotée car la population est plus dense et il existe des zones de banlieues sous-médicalisées. $\varepsilon n$ revanche, la densité départementale en médecins généralistes montre que des régions comme la Picardie, la Haute-Normandie, la Meuse, le Cotentin sont les moins bien dotées: moins de 142 médecins pour 100000 habitants. L'URCAM (union régionale des caisses d'assurance maladie) de Picardie a fait une étude détaillée de la démographie médicale [5] et a proposé en 2006 des avantages et des aides à l'installation des médecins dans les zones géographiques déficitaires (Figure 3).

\section{Les déserts médicaux}

Au sein des régions où la densité médicale est faible, on observe des zones encore plus défavorisées où la population ne peut avoir que difficilement accès à un médecin généraliste. Sur un département, la répartition est hétérogène, la répartition cantonale mettant à jour des graves déficits dans certains villages ruraux. Il s'agit généralement de régions qui se dépeuplent progressivement et dont la population qui vieillit n'en a pas moins besoin d'avoir recours à un médecin Sur l'ensemble du territoire, elle correspond à environ 4 \% (Figure 4).

\section{Désormais le point est fait}

L'ensemble des organisations concernées, très conscientes de la gravité du problème, ont établi des études statistiques et il est maintenant possible de trouver des renseignements suivis auprès du CNOM (conseil national de l'ordre des médecins), de la CNAMTS, de la CARMF avec exploitation ADELI (Association pour la Maîtrise des Systèmes d'Information).

De nombreuses études sont mises à jour régulièrement. Des outils en ligne d'aide à l'installation mis en place par la caisse primaire d'assurance maladie sont désormais accessibles sur les sites c@rtoSanté et inst@Isanté

Le rapport Berland, demandé par le ministre JeanFrançois Mattei en 2002, a fait des propositions très concrètes pour remédier aux «déserts médicaux » et fait l'objet d'un suivi [6].

\section{Comment remédier à cette situation?}

\section{Les moyens de régulation}

La gravité de cette situation a été soulignée en 2002 par Jean-François Mattei, alors Ministre de la Santé, de la Famille et des personnes handicapées, et par tous les autres ministres qui se sont succédés. Elle a donné lieu à des études statistiques régulièrement remises à jour de la part des DRESS, de I'URCARM, du conseil de l'Ordre des médecins. Mais aucune concertation ne s'est faite entre les différents partenaires.

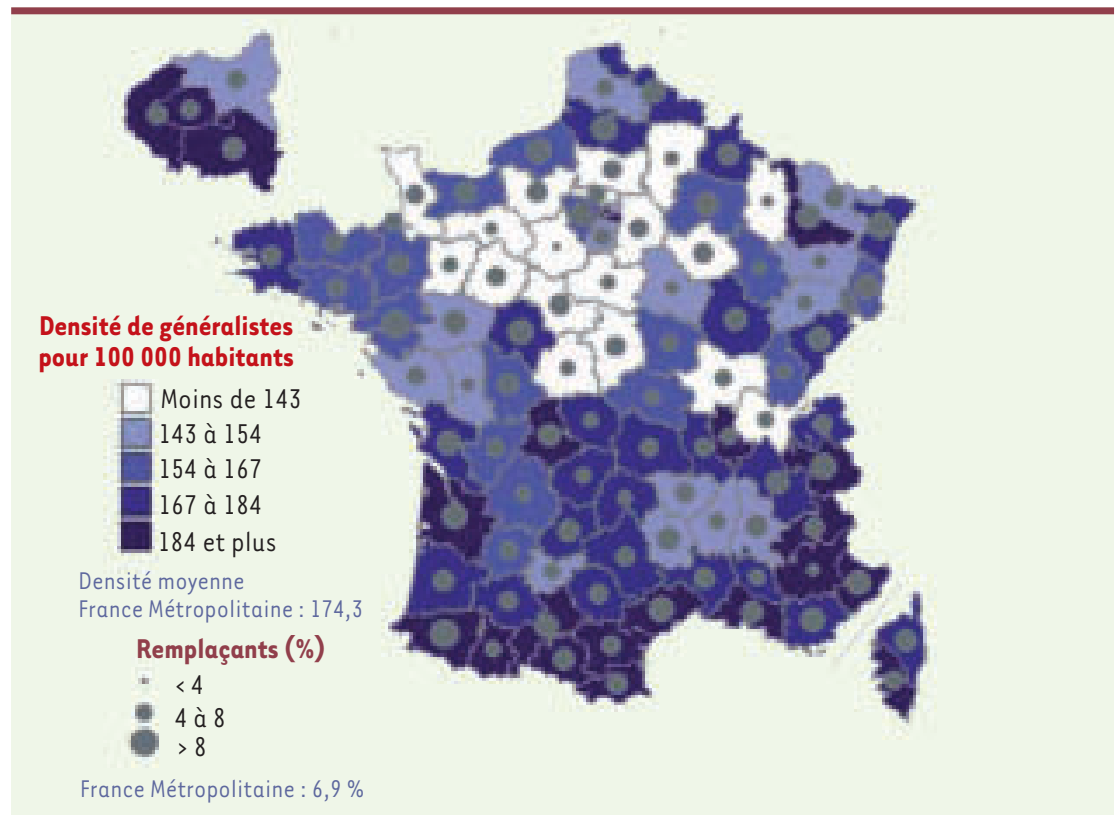

Figure 2. Densité départementale de médecins généralistes en activité professionnelle pour 100000 habitants en métropole (source CNOM 2005 : Ordre National des Médecins).

\section{Des solutions régionales} ou ponctuelles ont été proposées. Les MRS (Missions régionales de santé), I'ARH (Agence régionale d'hospitalisation) et les URCAM (Unions régionales des caisses d'assurance-maladie) s'engagent ensemble à l'amélioration des soins.

Elles proposent :

Des avantages financiers pour rendre la médecine générale attractive

Dans certaines régions, il existe des aides à l'installation.

- Majoration de $20 \%$ des honoraires dans le cadre de l'exercice collectif, à négocier avec les partenaires conventionnels.

- Financement du mode d'exercice en groupe offert par le FADSV (fond d'aide à la qualité des soins de ville).

- Des collectivités locales (conseils régionaux) proposent des bourses de fidélisation (300€/mois) durant les études, à 
condition de s'engager à exercer en milieu rural pendant une période d'au moins 5 ans.

La formation se fait dans les grandes villes, y compris les stages de 6 mois en cabinet. Des exemples démontrent que des stages en milieu rural, en particulier dans des maisons médicales, peuvent attirer des jeunes médecins.

- Des indemnités de logement et de déplacement sont proposées aux étudiants qui effectuent leur stage en zone rurale.

- Des avantages aux remplaçants : $300 €$ /jour dans la limite de 10 jours après signature d'un contrat de bonne pratique.

- Exonération partielle de l'impôt sur le revenu, et exonération de taxes professionnelles, ainsi qu'une autorisation d'un exercice en cabinet secondaire (décret du 17 mai 2005 modifiant le code de déontologie médicale).

Des solutions drastiques:

réforme fondamentale des études médicales

La réforme des $\varepsilon C N$ (épreuves classantes nationales) pourrait être remplacées par des $\varepsilon C R$ (épreuves classantes régionales) car nombre d'étudiants sont attachés à une région par leur famille et accepteraient peut-être de s'y installer.

Actuellement la répartition entre internes de spécialité et de médecine générale est de $60 \%-40 \%$, et il faudrait obtenir une répartition $50 \%-50 \%$.

Le problème des déserts médicaux est en réalité un phénomène de société beaucoup plus vaste. II touche aussi les petits hôpitaux qui

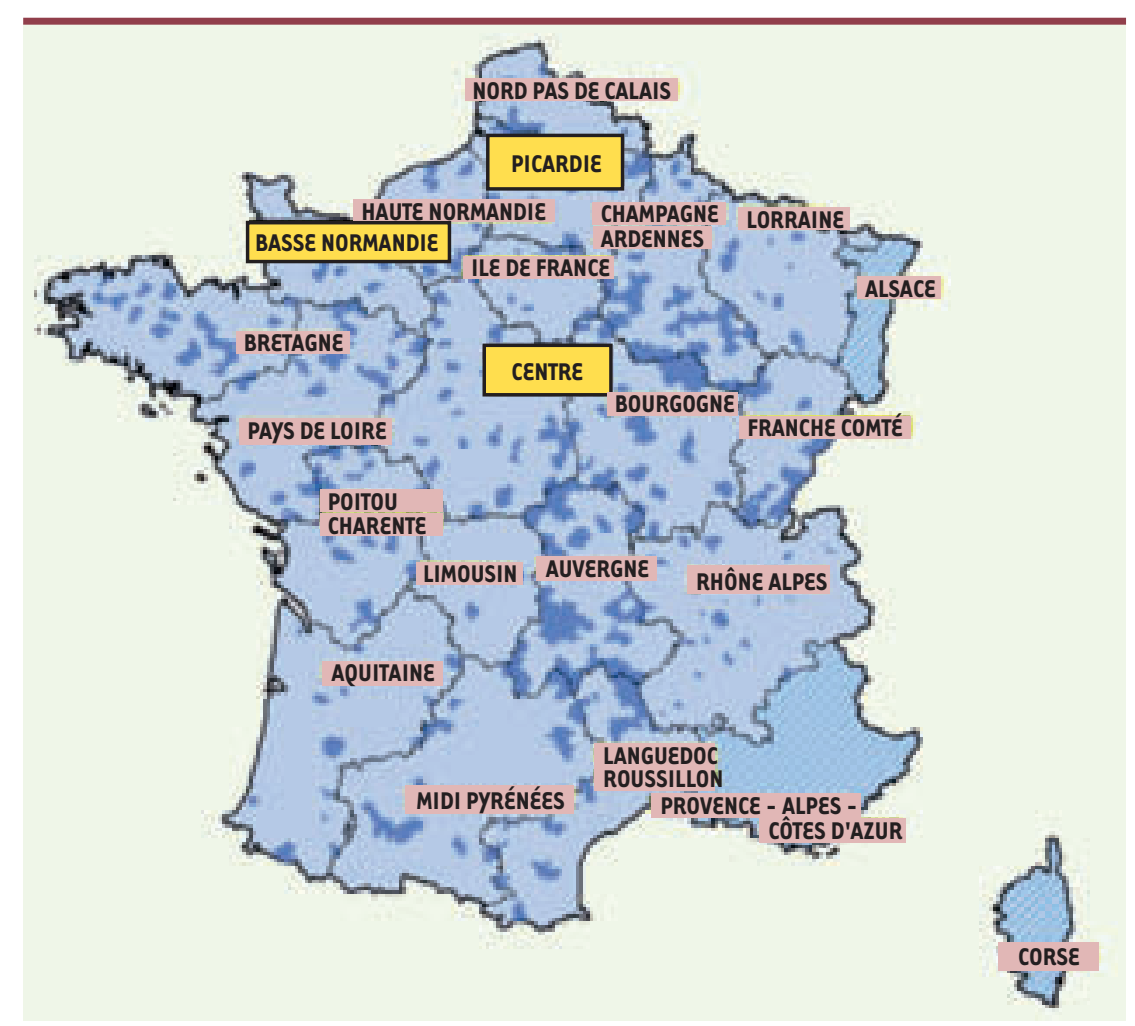

Figure 4. Régions ayant les densités les plus faibles avec mention des communes à faible densité médicales. Nom des régions en rose ; exemple de régions ayant une densité médicale parmi les plus faibles en jaune; zones à faible densité médicale en bleu foncé ; régions sans communes à faibles densité médicale en hachuré (source: DGS/bureau des systèmes d'information et CNOM 2005). éprouvent des difficultés à trouver des chirurgiens et des anesthésistes. Il touche les infirmiers et les métiers paramédicaux.

Une organisation liant les petits hôpitaux, les cliniques privées et les médecins avec un système de garde et une structure voisine de l'hôpital, ou au sein même de I'hôpital qui offrirait son plateau technique, permettrait une meilleure organisation des soins en même temps qu'une activité plus équilibrée. Elle est déjà prévue avec une convention entre les CHG (Centres hospitaliers généraux) et les petits hôpitaux locaux, qui facilite l'accès à des consultations et renforce la présence médicale.

La rémunération pourrait provenir de fonds publics: une enquête auprès des étudiants montre qu'ils sont beaucoup moins attachés que leurs aînés à la rémunération à l'acte.

\section{Réformer les statuts des médecins}

La création d'une charge médicale qui serait acquise au départ, comme les charges de notaires, et qui pourrait être revendue ultérieurement, - sauf si les postes sont déjà trop nombreux dans la région - représenterait une évolution avec une répartition plus équilibrée, à l'instar des installations des pharmaciens qui est réglementée dans ce sens. Mais, dans cette hypothèse, il faudrait que les médecins puissent revendre cette charge, ou que l'achat initial soit largement subventionné.

\section{Créer un métier intermédiaire entre} médecins et infirmiers

Proposé par le rapport Berland, un grade intermédiaire pourrait pallier le manque de médecins. Des expérimentations sont en cours visant à revaloriser les professions paramédicales, revue par la HAS (Haute Autorité de Santé) avec redéfinition des compétences: actes effectués par le personnel infirmier, et actes de spécialistes effectués par le médecin généraliste (gynécologie en particulier).

\section{Des solutions drastiques avec mesures coercitives}

On peut envisager aussi un abandon du remboursement à l'acte et une impossibilité - comme pour les pharmaciens - de s'installer dans des régions déjà médicalement surpeuplées.

Le système anglais, longtemps très décrié, semble actuellement donner satisfaction, mais il a été fondé sur une structure très différente au départ, et les Français vivant en Grande-Bretagne ont encore parfois du mal à s'y adapter. II y a deux fois moins de 
médecins en Angleterre qu'en France. Mais ils fonctionnent essentiellement en cabinets de groupe avec une équipe importante de secrétaires, infirmières, sages-femmes. Le NHS (national health service) rémunère plus les médecins installés dans des zones avec forte densité de patients précaires et lourds et en zone faiblement peuplée, mais il rencontre cependant des difficultés à pourvoir des zones isolées.

En Allemagne, depuis 1992, un système de numerus clausus territorial s'applique à la médecine libérale : pour être conventionné avec l'assurance-maladie, le nouveau médecin allemand ne peut s'installer que dans les zones à densité médicale faible. Ce système conduit à une raréfaction des jeunes praticiens et par conséquent à un vieillissement du corps médical, même dans la médecine hospitalière, en principe non soumise au numerus clausus territorial. Non seulement il y a une baisse de l'attractivité des études médicales mais aussi une déperdition grandissante des étudiants en médecine. Beaucoup parmi ces derniers, à la fin de leur formation médicale, préfèrent choisir des carrières situées en dehors des systèmes de soins. La diminution du nombre des jeunes praticiens en médecine libérale s'accompagne de vacances de postes dans l'enceinte hospitalière. Le pays se tourne de plus en plus vers le recrutement de médecins formés à l'étranger, en particulier dans les pays de l'Est [7].

Cet exemple conduit à être prudent, car interdire aux médecins français de s'installer dans des régions surmédicalisées ne les conduira pas à s'installer en zone rurale ou en zone urbaine difficile. Du reste, les syndicats, et en particulier le syndicat des internes en médecine y sont nettement hostiles.

\section{Conclusions}

L'apparition de déserts médicaux reflète un problème de société bien plus profond. II importe de valoriser les régions, de les redynamiser, et de faciliter les transports collectifs. II importe de faire que les rêves écologiques deviennent réalités.

Un autre mode de pilotage du système de santé avec des Agences Régionales de Santé établissant localement un maillage de l'organisation des soins qui uniraient les petits hôpitaux, les cliniques et les praticiens aurait sans doute plus d'efficacité qu'une organisation centralisée telle que nous la connaissons aujourd'hui.

Les propositions sont nombreuses: mentionnons le récent livre de Claude Evin sur ce point [8]. Une réforme complète des études médicales avec régionalisation de l'examen classant apparaît souhaitable. Le temps du praticien isolé est révolu et la médecine de groupe doit être encouragée, avec des incitations économiques directes et fiscales afin de rendre plus attractive l'activité du généraliste. En date du 4 avril 2007, le Conseil des ministres a décidé de revaloriser la consultation de médecine générale en faisant passer le tarif à $22 €$ à partir du $1^{\text {er }}$ août 2007 et à $23 €$ en 2008 à la suite de l'accord signé par les partenaires conventionnels le 29 mars dernier. Le développement de la filière universitaire sera également essentiel pour une spécification complète de la médecine générale. Celle-ci devrait conférer aux étudiants une formation pratique leur permettant de faire de la médecine ambulatoire - ce dont ils sont incapables actuellement - et qui les inciterait peut-être à s'orienter vers la médecine générale. $\diamond$

Medical demography:

how to fight against the medical deserts?

\section{REMERCIEMENTS}

Nous remercions Gérard de Pouvourville pour ses conseils et son aide patiente et efficace.

\section{RÉFÉRENCES}

1. http://www.fmrq.qc.ca/formation-medicale/liensUtiles.cfm"regions

2. Flori F. La médecine générale en France : entre omnipratique et orientations complémentaires. Med Sci (Paris) 2001 ; 17 : 924-6.

3. http://www.sante.gouv.fr/htm/actu/berland/sommaire.htm

4. Sicart D. Les médecins. Estimations au $1^{\text {er }}$ janvier 2006. DREદS Études et Résultats octobre $2006 ; n^{\circ} 103$.

5. http://www.picardie.sante.gouv.fr/etudes.htm

6. www.sante.gouv.fr/htm/actu/berland2006/rapport.pdf

7. Kopetsch T. The medical profession in Germany: past trends, current state and future prospects. Cahiers de Sociologie et de Démographie Médicales $2004 ; 44: 43-70$.

8. Evin C, Legastelois J. À votre Santé ! Paris : Éditions de l'Atelier, 2006.

\section{POUR EN SAVOIR PLUS}

- Perriot G. J'étais médecin de campagne. Paris : AZ Patrimoine, 2006. - Bessière $S$, Breuil-Genier P, Darriné $S$. La démographie médicale à I’horizon 2025 : une actualisation des projets au niveau national. DREES Études et Résultats novembre 2004, $n^{\circ} 352$.

- Donzelot J. Quand la ville se défait : quelle politique face à la crise des banlieues? Paris : Seuil, 2006.

\section{TIRÉS À PART}

S. Gilgenkrantz

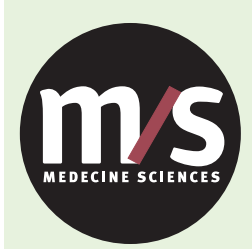

Tarifs d'abonnement M/S - 2007

Abonnez-vous

à Médecine/Sciences
> Depuis 20 ans, grâce à $m / s$, vous vivez en direct les progrès des sciences biologiques et médicales

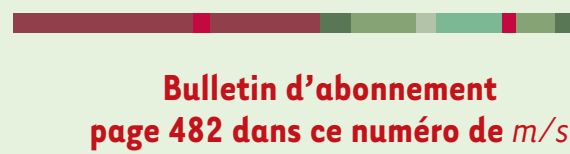

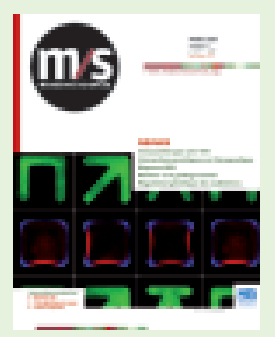

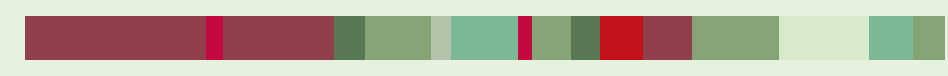

